\title{
Superpositions of Probability Distributions
}

\author{
Petr Jizba* and Hagen Kleinert ${ }^{\dagger}$ \\ ITP, Freie Universität Berlin \\ Arnimallee 14 D-14195 Berlin, Germany
}

(Dated: October 4, 2018)

\begin{abstract}
Probability distributions which can be obtained from superpositions of Gaussian distributions of different variances $v=\sigma^{2}$ play a favored role in quantum theory and financial markets. Such superpositions need not necessarily obey the Chapman-Kolmogorov semigroup relation for Markovian processes because they may introduce memory effects. We derive the general form of the smearing distributions in $v$ which do not destroy the semigroup property. The smearing technique has two immediate applications. It permits simplifying the system of Kramers-Moyal equations for smeared and unsmeared conditional probabilities, and can be conveniently implemented in the path integral calculus. In many cases, the superposition of path integrals can be evaluated much easier than the initial path integral. Three simple examples are presented, and it is shown how the technique is extended to quantum mechanics.

PACS numbers: 02.50.Ga, 05.10.Gg, 05.45.Tp, 05.40.-a
\end{abstract}

*Electronic address: jizba@physik.fu-berlin.de; On leave from

FNSPE, Czech Technical University, Břehová 7, 11519 Praha 1, Czech Republic

${ }^{\dagger}$ Electronic address: kleinert@physik.fu-berlin.de 


\section{INTRODUCTION}

Path integrals are a powerful tool in diverse areas of physics, both computationally and conceptually. They often provide the easiest route to derivation of perturbative expansions as well as an excellent framework for nonperturbative analysis [1]. One of the key properties of path integrals in statistical physics is that the related time evolution of the conditional probabilities $P\left(x_{b}, t_{b} \mid x_{a}, t_{a}\right)$ fulfills the Chapman-Kolmogorov (C-K) equation for continuous Markovian processes

$$
P\left(x_{b}, t_{b} \mid x_{a}, t_{a}\right)=\int \mathrm{d} x P\left(x_{b}, t_{b} \mid x, t\right) P\left(x, t \mid x_{a}, t_{a}\right)
$$

Conversely, any probability satisfying this equation possesses a path integral representation,

as shown by Kac and Feynman [2-4]. Equation (1) also serves as a basis for deriving a Fokker-Planck time evolution equation $[1,5,6]$ for $P\left(x_{b}, t_{b} \mid x_{a}, t_{a}\right)$ from either the stochastic differential equation obeyed by the variable $x$ or the Hamiltonian driving the time evolution of $P\left(x_{b}, t_{b} \mid x_{a}, t_{a}\right)$. Such equations are used to explain many different physical phenomena, for example turbulence [7] or epitaxial growth [8]. In information theory they serve as a tool for modeling various queueing processes [9], while in mathematical finance they are conveniently applied in theory of option pricing for efficient markets [1, 10-12].

A trivial property of $P\left(x_{b}, t_{b} \mid x_{a}, t_{a}\right)$ satisfying the $\mathrm{C}-\mathrm{K}$ equation (1) is the initial condition

$$
P\left(x_{b}, t_{a} \mid x_{a}, t_{a}\right)=\delta\left(x_{b}-x_{a}\right)
$$

The right-hand side can be written as a scalar product of Dirac's bra and ket states $\left\langle x_{b}\right|$ and $\left|x_{a}\right\rangle$ as

$$
P\left(x_{b}, t_{a} \mid x_{a}, t_{a}\right)=\left\langle x_{b} \mid x_{a}\right\rangle
$$

In many practical applications one encounters conditional probabilities formulated as a superposition of path integrals in which the Hamiltonians $H$ are rescaled by a factor $v$, i.e.,

$$
\bar{P}\left(x_{b}, t_{b} \mid x_{a}, t_{a}\right)=\int_{0}^{\infty} \mathrm{d} v \omega\left(v, t_{b a}\right) \int_{x\left(t_{a}\right)=x_{a}}^{x\left(t_{b}\right)=x_{b}} \mathcal{D} x \mathcal{D} p e^{\int_{t_{a}}^{t_{b}} \mathrm{~d} \tau[i p \dot{x}-v H(p, x)]}
$$

where $\omega\left(v, t_{b a}\right)$ is some positive, continuous and normalizable smearing function of $v \geq 0$ and $t_{b a} \equiv t_{b}-t_{a} \geq 0$. For instance, probability distributions which can be obtained from superpositions of Gaussian distributions of different volatilities $v=\sigma^{2}$ play an important 
role in financial markets $[1,10]$. Such smearing distributions show up also in nonperturbative approximations to quantum statistical partition functions [13], in systems with time reparametrization invariance $[1,14]$, in polymer physics $[1,15]$, in superstatistics [16], etc. Whenever smeared path integrals fulfill the C-K equation, the Feynman-Kac formula ensures that such superpositions can themselves be written as a path integral without smearing

$$
\bar{P}\left(x_{b}, t_{b} \mid x_{a}, t_{a}\right)=\int_{x\left(t_{a}\right)=x_{a}}^{x\left(t_{b}\right)=x_{b}} \mathcal{D} x \mathcal{D} p e^{\int_{t_{a}}^{t_{b}} \mathrm{~d} \tau[i p \dot{x}-\bar{H}(p, x)]},
$$

with a new Hamiltonian $\bar{H}(p, x)$ given by

$$
e^{-\int_{t_{a}}^{t_{b}} \mathrm{~d} \tau \bar{H}(p, x)}=\int_{0}^{\infty} \mathrm{d} v \omega\left(v, t_{b a}\right) e^{-\int_{t_{a}}^{t_{b}} \mathrm{~d} \tau v H(p, x)}
$$

In general, the smeared path integral (4) does not conserve the C-K equation. Physically this implies that the superposition (4) introduces memory into the system. The aim of this note is to find conditions for the smearing distributions where this is avoided. Various physical consequences will be derived from this.

The paper is organized as follows. Section II starts with a warm up example of smearing distributions for a Gaussian conditional probability. In Section III we derive the most general class of continuous smearing distributions fulfilling C-K equation. Section IV is devoted to a construction of the Hamiltonian $\bar{H}$ and to a discussion of related issues. In Section $\mathrm{V}$ we show how the outlined path integral representation can be physically interpreted in terms of two coupled stochastic processes. Section VI discusses three specific smeared systems without memory. In particular we show how the explicit knowledge of $\bar{H}(p, x)$ can streamline practical calculations of numerous path integrals. Various remarks and generalizations are proposed in the concluding Section VII. For reader's convenience we include two appendices where we perform some finer mathematical manipulations needed in Section V.

\section{SMEARING OF A GAUSSIAN DISTRIBUTION}

Our goal is to find the most general form of $\omega(v, t)$ fulfilling the $\mathrm{C}-\mathrm{K}$ relation (1). Let us first illustrate what we want to achieve by smearing out a simple Gaussian system whose 
Hamiltonian is $H=v p^{2} / 2$ leading to a conditional probability

$$
\begin{aligned}
P_{v}\left(x_{b}, t_{b} \mid x_{a}, t_{a}\right) & =\int_{x\left(t_{a}\right)=x_{a}}^{x\left(t_{b}\right)=x_{b}} \mathcal{D} x \mathcal{D} p e^{\int_{t_{a}}^{t_{b}} \mathrm{~d} \tau\left[i p \dot{x}-v p^{2} / 2\right]} \\
& =\frac{1}{\sqrt{2 \pi v t_{b a}}} e^{-\left(x_{b}-x_{a}\right)^{2} /\left(2 v t_{b a}\right)},
\end{aligned}
$$

where $t_{b a} \equiv t_{b}-t_{a}$. This obeys the Fokker-Planck equation

$$
\partial_{t_{b}} P_{v}\left(x_{b}, t_{b} \mid x_{a}, t_{a}\right)=\frac{v}{2} \partial_{x_{b}}^{2} P_{v}\left(x_{b}, t_{b} \mid x_{a}, t_{a}\right) .
$$

which can be solved explicitly, including the initial condition (2) with the help of the differential operator for the momentum $\hat{p} \equiv-i \partial_{x}$ as

$$
P_{v}\left(x_{b}, t_{b} \mid x_{a}, t_{a}\right)=e^{-t_{b a} v \hat{p}^{2} / 2} \delta\left(x_{b}-x_{a}\right),
$$

or, using the Dirac bra and ket states in (3), as

$$
P_{v}\left(x_{b}, t_{b} \mid x_{a}, t_{a}\right)=\left\langle x_{b}\left|e^{-t_{b a} v \hat{p}^{2} / 2}\right| x_{a}\right\rangle
$$

Due to the completeness relation $\int \mathrm{d} x|x\rangle\langle x|=1$, this expression obviously satisfies the C-K equation (1).

Let us now assume that $\omega(v, t)$ can be written as a Fourier transform of the form [compare also Ref. [17]]:

$$
\omega\left(v, t_{b a}\right)=\int_{-i \infty}^{i \infty} \frac{\mathrm{d} \xi}{2 \pi i} e^{\xi v-H_{\omega}\left(\xi / t_{b a}\right) t_{b a}}
$$

where $H_{\omega}(\xi)$ can be viewed as the Hamiltonian affiliated with distribution $\omega$. Then the smeared transition probability has the integral representation

$$
\bar{P}\left(x_{b}, t_{b} \mid x_{a}, t_{a}\right)=\int_{-i \infty}^{i \infty} \frac{\mathrm{d} \xi}{2 \pi i}\left\langle x_{b}\left|\frac{e^{-H_{\omega}\left(\xi / t_{b a}\right) t_{b a}}}{\xi-t_{b a} \hat{p}^{2} / 2}\right| x_{a}\right\rangle .
$$

Assuming that $H_{\omega}(\xi)$ is a regular function which becomes infinite on a large semicircle in the upper half plane we can use the residue theorem to evaluate this as

$$
\bar{P}\left(x_{b}, t_{b} \mid x_{a}, t_{a}\right)=\left\langle x_{b}\left|e^{-H_{\omega}\left(i \hat{p}^{2} / 2\right) t_{b a}}\right| x_{a}\right\rangle
$$

This can be written as a path integral

$$
\bar{P}\left(x_{b}, t_{b} \mid x_{a}, t_{a}\right)=\int_{x\left(t_{a}\right)=x_{a}}^{x\left(t_{b}\right)=x_{b}} \mathcal{D} x \mathcal{D} p e^{\int_{t a}^{t_{b}} \mathrm{~d} \tau\left[i p \dot{x}-H_{\omega}\left(p^{2} / 2\right)\right]} .
$$


Thus the Hamiltonian $\bar{H}(p, x)$ of the smeared system in Eq. (5) is simply equal to $H_{\omega}\left(p^{2} / 2\right)$.

In the following we shall generalize this treatment to non-Gaussian Hamiltonians in Eq. (7).

\section{SMEARING OF A GENERAL DISTRIBUTION}

We now embark on finding the most general smearing function $\omega\left(v, t_{a b}\right)$ to guarantee the C-K relation for the smeared expression (4). Replacing the probabilities in (1) by (4), we bring the right-hand side to the explicit form

$$
\begin{gathered}
\int_{0}^{\infty} \mathrm{d} v^{\prime} \omega\left(v^{\prime}, t^{\prime}\right) \int_{0}^{\infty} \mathrm{d} v^{\prime \prime} \omega\left(v^{\prime \prime}, t^{\prime \prime}\right) \int_{-\infty}^{\infty} \mathrm{d} x \int_{x\left(t_{c}\right)=x}^{x\left(t_{b}\right)=x_{b}} \mathcal{D} x \mathcal{D} p e^{\int_{t_{c}}^{t_{b}} \mathrm{~d} \tau\left(i p \dot{x}-v^{\prime \prime} H\right)} \\
\times \int_{x\left(t_{a}\right)=x_{a}}^{x\left(t_{c}\right)=x} \mathcal{D} x \mathcal{D} p e^{\int_{t_{a}}^{t_{c}} \mathrm{~d} \tau\left(i p \dot{x}-v^{\prime} H\right)} \\
=\frac{t}{2 t^{\prime} t^{\prime \prime}} \int_{0}^{\infty} \mathrm{d} v \int_{-t v}^{t v} \mathrm{~d} \zeta \omega\left(\frac{(t v-\zeta)}{2 t^{\prime}}, t^{\prime}\right) \omega\left(\frac{(t v+\zeta)}{2 t^{\prime \prime}}, t^{\prime \prime}\right) \int_{x\left(t_{a}\right)=x_{a}}^{x\left(t_{b}\right)=x_{b}} \mathcal{D} x \mathcal{D} p e^{\int_{t_{a}}^{t_{b}} \mathrm{~d} \tau(i p \dot{x}-v H)},
\end{gathered}
$$

where $t^{\prime} \equiv t_{c a}=t_{c}-t_{a}$ and $t^{\prime \prime} \equiv t_{b c}=t_{b}-t_{c}$. In the second line we have used the substitution $t^{\prime \prime} v^{\prime \prime}+t^{\prime} v^{\prime}=t v$ and $t^{\prime \prime} v^{\prime \prime}-t^{\prime} v^{\prime}=\zeta$. Comparing the right-hand side of (15) with the left-hand side of (4) expressed in the smeared form (4), we obtain an integral equation for the smearing function $\omega(v, t)$ :

$$
\int_{-t v}^{t v} \mathrm{~d} \zeta \omega\left(\frac{(t v-\zeta)}{2 t^{\prime}}, t^{\prime}\right) \omega\left(\frac{(t v+\zeta)}{2 t^{\prime \prime}}, t^{\prime \prime}\right)=\frac{2 t^{\prime} t^{\prime \prime} \omega(v, t)}{t} .
$$

Setting $z \equiv t v / t^{\prime \prime}$ and going over to an integration variable $z^{\prime}=(t v+\zeta) / 2 t^{\prime \prime}$, this becomes

$$
\int_{0}^{z} d z^{\prime} \omega\left(z^{\prime}, t^{\prime \prime}\right) \omega\left(\frac{t^{\prime \prime}}{t^{\prime}}\left(z-z^{\prime}\right), t^{\prime}\right)=\frac{t^{\prime}}{t} \omega\left(\frac{t^{\prime \prime}}{t} z, t\right),
$$

or equivalently

$$
\int_{0}^{z} d z^{\prime} \omega\left(z^{\prime}, t\right) a \omega\left(a\left(z-z^{\prime}\right), \frac{t}{a}\right)=b \omega\left(b z, \frac{t}{b}\right),
$$

with positive real $a, b$ satisfying $1+1 / a=1 / b$. Since the left-hand side is a convolution integral, the solution of this equation is found by a Laplace transformation. Defining

$$
\tilde{\omega}(\xi, t)=\int_{0}^{\infty} \mathrm{d} z e^{-\xi z} \omega(z, t), \quad \operatorname{Re} \xi>0,
$$


we may reduce Eq. (18) to the functional equation

$$
\tilde{\omega}(\xi, t) \tilde{\omega}\left(\frac{\xi}{a}, \frac{t}{a}\right)=\tilde{\omega}\left(\frac{\xi}{b}, \frac{t}{b}\right) .
$$

It should be stressed that due to assumed normalizability and positivity of $\omega(v, t)$, the smearing distribution is always Laplace transformable. The substitution $\alpha=1 / a$ transforms (20) to

$$
\tilde{\omega}(\xi, t) \tilde{\omega}(\alpha \xi, \alpha t)=\tilde{\omega}(\xi+\alpha \xi, t+\alpha t) .
$$

By considering only real $\xi$, Eq. (21) can be solved by method of iterations familiar from the theory of functional equations [18]. Assume for a moment that $\alpha$ is a positive integer, say $n$, then successive iterations of Eq. (21) give

$$
\tilde{\omega}(n \xi, n t)=[\tilde{\omega}(\xi, t)]^{n} .
$$

Now let $r=m / n$ be a positive rational number ( $m$ and $n$ positive integers) and $\zeta$ and $\tau$ arbitrary positive real numbers. Then, for $\xi=r \zeta=(m / n) \zeta$ and $t=r \tau=(m / n) \tau$, we have $n \xi=m \zeta$ and $n t=m \tau$, so that Eq. (22) yields

$$
\tilde{\omega}(\xi, t)=\tilde{\omega}(r \zeta, r \tau)=[\tilde{\omega}(\zeta, \tau)]^{r}
$$

for all positive $\zeta$ and $\tau$ and all positive rationals $r$. Assuming that $\tilde{\omega}$ is continuous, we may extend the Eq. (23) to all positive real $r$. It is then solved by

$$
\begin{aligned}
\tilde{\omega}(\xi, t) & =\tilde{\omega}(t \cdot \xi / t, t \cdot 1) \\
& =[\tilde{\omega}(\xi / t, 1)]^{t} \equiv[G(\xi / t)]^{t}, \quad t>0,
\end{aligned}
$$

where $G(x)$ is any continuous function of $x$. The above derivation is meaningless for $t=0$. In this case we must instead of (24) consider

$$
\tilde{\omega}(\xi, 0)=\tilde{\omega}(\xi \cdot 1, \xi \cdot 0)=[\tilde{\omega}(1,0)]^{\xi} \equiv \kappa^{\xi} .
$$

The constant $\kappa$ is determined by the initial value of the smearing distribution $\omega(v, t)$. Thus Eq. (25) implies that

$$
\begin{aligned}
\lim _{t \rightarrow+0} \omega(v, t) & =\theta(v+\log \kappa) \delta(v+\log \kappa) \\
& =\delta^{+}(v+\log \kappa) .
\end{aligned}
$$


Note that Eq. (24) implies positivity of $\tilde{\omega}(\xi, t)$ for all $t$ and $\xi$, hence $G(x)$ must also be positive for all $x$. This allows us to write

$$
[G(\xi / t)]^{t}=e^{-F(\xi / t) t}
$$

where $F(x)$ is some continuous function of $x$. The associated inverse Laplace transform gives then the complete solution for $\omega(v, t)$. Usually, the inverse Laplace transform is expressed as as a complex Bromwich integral [19] [compare (11)]:

$$
\omega(v, t)=\frac{1}{2 \pi i} \int_{\gamma-i \infty}^{\gamma+i \infty} \mathrm{d} \xi e^{\xi v} \tilde{\omega}(\xi, t),
$$

where the real constant $\gamma$ is such that it exceeds the real part of all the singularities of $\tilde{\omega}(\xi, t)$. For our purpose it will be preferable to use, instead of (28), the inversion formula due to Post [20]:

$$
\omega(v, t)=\left.\lim _{k \rightarrow \infty} \frac{(-1)^{k}}{k !}\left(\frac{k}{v}\right)^{k+1} \partial_{\xi}^{k} \tilde{\omega}(\xi, t)\right|_{\xi=k / v} .
$$

For practical calculations this formula is rarely used due to the need to evaluate derivatives of arbitrary high orders. For our purpose, however, it has the advantage that it shows that a real Laplace transform $\tilde{\omega}(\xi, t)$ leads to a real smearing function $\omega\left(v, t_{a b}\right)$. Moreover, one does not need to know the pole structure of $\tilde{\omega}(\xi, t)$ in the complex $\xi$-plane, which is in general not known.

The result (24) can be mildly generalized to the smearing of composite Hamiltonians $v H(p, x)=v H_{1}(p, x)+H_{2}(p, x)$ as long as $\left[\hat{H}_{1}, \hat{H}_{2}\right]=0$. At the same time it should be stressed that the entire approach fails for time-dependent Hamiltonians.

To end this section we note that if the smearing distribution $\omega(v, t)$ would have included negative $v$-values, the integral in Eq. (18) would have been replaced with $\int_{0}^{z} \mapsto \int_{\infty}^{-\infty}$. Then a two-sided Laplace transformation would have brought us to the same equations (23)-(24) as before, and the associated general $\omega(v, t)$ would been recovered via the inverse of the two-sided Laplace transform. By restricting ourselves to $v \geq 0$, all calculations become simpler. 


\section{EXPLICIT REPRESENTATION OF $\bar{H}(p, x)$}

We now determine the Hamiltonian $\bar{H}(p, x)$ explicitly in the general case. For this we must first make sure that the path integral for the initial distribution in (4)

$$
P_{v}\left(x_{b}, t_{b} \mid x_{a}, t_{a}\right)=\int_{x\left(t_{a}\right)=x_{a}}^{x\left(t_{b}\right)=x_{b}} \mathcal{D} x \mathcal{D} p e^{\int_{t_{a}}^{t_{b}} \mathrm{~d} \tau[i p \dot{x}-v H(p, x)]}
$$

is properly defined. The classical Hamiltonian $H(p, x)$ must be set up in such a way that the Hamiltonian operator driving the time evolution

$$
\partial_{t} P_{v}\left(x, t \mid x_{a}, t_{a}\right)=-v \hat{H}(\hat{p}, x) P_{v}\left(x, t \mid x_{a}, t_{a}\right),
$$

has all momentum operators $\hat{p}=-i \partial_{x}$ to the left of the $x$-variables. Only then can one guarantee the probability conservation law for $P\left(x_{b}, t_{b} \mid x_{a}, t_{a}\right)$ :

$$
\int \mathrm{d} x \partial_{t} P_{v}\left(x, t \mid x_{a}, t_{a}\right)=-\int \mathrm{d} x v \hat{H}(\hat{p}, x) P_{v}\left(x, t \mid x_{a}, t_{a}\right) .
$$

The right-hand side vanishes after a partial integration. The relation between $\hat{H}(\hat{p}, x)$ and $H(p, x)$ is explained in Ref. [1].

With the help of Post's inversion formula (29) we now rewrite the smeared conditional probability (4) in the form

$$
\begin{aligned}
\bar{P}\left(x_{b}, t_{b} ; x_{a}, t_{a}\right) & =\lim _{k \rightarrow \infty} \frac{(-1)^{k-1}}{(k-1) !} \int_{0}^{\infty} \mathrm{d} \xi \xi^{k-1} \partial_{\xi}^{k} \tilde{\omega}\left(\xi, t_{b a}\right) \\
& \times \int_{x\left(t_{a}\right)=x_{a}}^{x\left(t_{b}\right)=x_{b}} \mathcal{D} x \mathcal{D} p e^{\int_{t a}^{t_{b}} \mathrm{~d} \tau(i p \dot{x}-k H / \xi)}
\end{aligned}
$$

Inserting here Eq. (19), the integration over $\xi$ turns into

$$
\int_{0}^{\infty} \mathrm{d} v v^{k} \omega\left(v, t_{b a}\right) \int_{0}^{\infty} \mathrm{d} \xi \xi^{k-1} e^{-v \xi} \int_{x\left(t_{a}\right)=x_{a}}^{x\left(t_{b}\right)=x_{b}} \mathcal{D} x \mathcal{D} p e^{\int_{t_{a}}^{t_{b}} \mathrm{~d} \tau[i p \dot{x}-k H / \xi]} .
$$

The path integral can be written in Dirac operator form (10) as

$$
\left\langle x_{b}\left|e^{-k t_{b a} \hat{H} / \xi}\right| x_{a}\right\rangle
$$

so that (34) becomes

$$
\int_{0}^{\infty} \mathrm{d} v v^{k} \omega\left(v, t_{b a}\right)\left\langle x_{b}\left|\int_{0}^{\infty} \mathrm{d} \xi \xi^{k-1} e^{-v \xi} e^{-k t_{b a} \hat{H} / \xi}\right| x_{a}\right\rangle
$$


The $\xi$-integral yields a Bessel function of the second type

$$
2\left(k t_{b a} \hat{H} / y\right)^{k / 2} K_{k}\left(2 \sqrt{k y t_{b a} \hat{H}}\right) .
$$

Applying the limiting form of the Bessel function for large index

$$
K_{k}(\sqrt{k} x) \sim \frac{1}{2} k^{-k / 2} \Gamma(k)\left(\frac{x}{2}\right)^{-k} e^{-x^{2} / 4},
$$

we can cast (33) to

$$
\bar{P}\left(x_{b}, t_{b} \mid x_{a}, t_{a}\right)=\left\langle x_{b}\left|\int_{0}^{\infty} \mathrm{d} v \omega\left(v, t_{b a}\right) e^{-v t_{b a} \hat{H}}\right| x_{a}\right\rangle .
$$

We now use Eqs. (19) and (24) to rewrite this as

$$
\left\langle x_{b}\left|\tilde{\omega}\left(t_{b a} \hat{H}, t_{b a}\right)\right| x_{a}\right\rangle=\left\langle x_{b}\left|\tilde{\omega}(\hat{H}, 1)^{t_{b a}}\right| x_{a}\right\rangle .
$$

With Eq. (27), this becomes

$$
\bar{P}\left(x_{b}, t_{b} \mid x_{a}, t_{a}\right)=\left\langle x_{b}\left|e^{-t_{b a} F(\hat{H})}\right| x_{a}\right\rangle,
$$

which can be expressed as a path integral

$$
\bar{P}\left(x_{b}, t_{b} \mid x_{a}, t_{a}\right)=\int_{x\left(t_{a}\right)=x_{a}}^{x\left(t_{b}\right)=x_{b}} \mathcal{D} x \mathcal{D} p e^{\int_{t_{a}}^{t_{b}} \mathrm{~d} t\left[i p \dot{x}-F_{\mathrm{cl}}(H)\right]} .
$$

Here $F_{\mathrm{cl}}(H)$ denotes the classical function of the energy which makes the path integral (42) equal to the operator expression $F(\hat{H})$ in (41). The construction of (42) is highly nontrivial since one must ensure that the path integral leads to the correct operator order in $\tilde{\omega}(\hat{H}, 1)^{t_{b a}}$. The task is simple only if $\hat{H}$ depends only on $\hat{p}$. Then the ordering problem disappears and $F_{\mathrm{cl}}(H(p))=F(H(p))$.

There are other instances in which the operator ordering can be uniquely assigned. In Appendix B we discuss one such example.

A few observations are useful concerning he nature of $F(H)$. First, the normalization condition

$$
1=\int_{0}^{\infty} \mathrm{d} v \omega(v, t)=\tilde{\omega}(0, t),
$$

implies that $F(0)=0$. Second, the necessary positivity of $\omega(v, t)$ implies, via Post's formula, that all derivatives $(-1)^{k} \partial^{k} \tilde{\omega}(\xi, t) / \partial \xi^{k}$ are positive for large $\xi$ and $k$. Taking into account 
that $\tilde{\omega}(x, t)>0$ we conclude that $\tilde{\omega}(\xi, t)$ must be decreasing and convex for large $\xi$ and any $t$. Asymptotic decrease and convexity of $e^{-F(x / t) t}$ ensure that $F(\hat{H})$ must be a monotonically increasing function of $\hat{H}$ for large spectral values of $\hat{H}$. Third, the real nature of $\tilde{\omega}(x, t)$ makes $F(\hat{H})$ a real function of $\hat{H}$. This is in contrast to quantum-mechanical path integrals where the smearing function $\omega(v, t)$ is not necessarily real.

\section{KRAMERS-MOYAL EXPANSIONS}

Let us study the implications of the above smearing procedure upon the time evolution equations for the conditional probabilities $P_{v}\left(x_{b}, t_{b} \mid x_{a}, t_{a}\right)$ and $\bar{P}\left(x_{b}, t_{b} \mid x_{a}, t_{a}\right)$ which have the general form (31). In statistical physics these are called Kramers-Moyal (K-M) equations [1, $6,23]$. The negative time evolution operator $-v \hat{H}(\hat{p}, x)$ is called Kramers-Moyal operators $\mathbb{L}_{v}\left(-\partial_{x}, x\right)$. Thus Eq. (31) for $P_{v}\left(x_{b}, t_{b} \mid x_{a}, t_{a}\right)$ and an analogous equation for $\bar{P}\left(x_{b}, t_{b} \mid x_{a}, t_{a}\right)$ are written as

$$
\begin{aligned}
\partial_{t_{b}} P_{v}\left(x_{b}, t_{b} \mid x_{a}, t_{a}\right) & =\mathbb{L}_{v} P_{v}\left(x_{b}, t_{b} \mid x_{a}, t_{a}\right), \\
\partial_{t_{b}} \bar{P}\left(x_{b}, t_{b} \mid x_{a}, t_{a}\right) & =\overline{\mathbb{L}} \bar{P}\left(x_{b}, t_{b} \mid x_{a}, t_{a}\right) .
\end{aligned}
$$

The Kramers-Moyal operator $\mathbb{L}_{v}$ has the expansion

$$
\mathbb{L}_{v}\left(-\partial_{x_{b}}, x_{b}\right)=\sum_{n=1}^{\infty}\left(-\partial_{x_{b}}\right)^{n} D_{v}^{(n)}\left(x_{b}, t_{b}\right),
$$

whose coefficients $D_{v}^{(n)}(x, t)$ are equal to the moments of the short-time transition probabilities:

$$
D_{v}^{(n)}(x, t)=\frac{1}{n !} \lim _{\tau \rightarrow 0} \frac{1}{\tau} \int_{-\infty}^{\infty} \mathrm{d} y(y-x)^{n} P_{v}(y, t+\tau \mid x, t) .
$$

Inserting Eq. (31), these can also be calculated from the formula

$$
D_{v}^{(n)}(x, t)=\frac{1}{n !} \lim _{\tau \rightarrow 0} \frac{1}{\tau} \int_{-\infty}^{\infty} \mathrm{d} y(y-x)^{n}\left\langle y\left|e^{-v \hat{H} \tau}\right| x\right\rangle .
$$

The same equation holds for $\bar{P}\left(x_{b}, t_{b} \mid x_{a}, t_{a}\right)$ with the replacement $v \hat{H} \rightarrow \hat{\bar{H}}, \mathbb{L}_{v} \rightarrow \overline{\mathbb{L}}$, and $D_{v}^{(n)}(x, t) \rightarrow \bar{D}^{(n)}(x, t)$.

Our smearing procedure can be recast in the language of K-M equations. We show that the two equations (44) and (45) can be replaced by an equivalent pair of K-M equations. 
First we observe that Eq. (17) can be rewritten as

$$
\omega(z, t)=\int_{0}^{\infty} \mathrm{d} z^{\prime} P_{\omega}\left(z, t \mid z^{\prime}, t^{\prime}\right) \omega\left(z^{\prime}, t^{\prime}\right),
$$

with the conditional probability

$$
P_{\omega}\left(z, t \mid z^{\prime}, t^{\prime}\right)=\frac{t}{t-t^{\prime}} \theta\left(t z-t^{\prime} z^{\prime}\right) \omega\left(\frac{t z-t^{\prime} z^{\prime}}{t-t^{\prime}}, t-t^{\prime}\right)
$$

which satisfies the initial condition

$$
\lim _{\tau \rightarrow 0} P_{\omega}\left(z, t+\tau \mid z^{\prime}, t\right)=\delta^{+}\left(z-z^{\prime}\right)
$$

Moreover, expressing $\omega(z, t)$ in Eq. (49) in terms of $P_{\omega}\left(x_{b}, t_{b} \mid x_{a}, t_{a}\right)$ via (50), we find that $P_{\omega}\left(x_{b}, t_{b} \mid x_{a}, t_{a}\right)$ satisfies a $\mathrm{C}-\mathrm{K}$ equation

$$
P_{\omega}\left(x_{b}, t_{b} \mid x_{a}, t_{a}\right)=\int \mathrm{d} x P_{\omega}\left(x_{b}, t_{b} \mid x, t\right) P_{\omega}\left(x, t \mid x_{a}, t_{a}\right) .
$$

This implies that $P_{\omega}\left(x_{b}, t_{b} \mid x_{a}, t_{a}\right)$ obeys a K-M time evolution equation

$$
\partial_{t_{b a}} P_{\omega}\left(v_{b}, t_{b} \mid v_{a}, t_{a}\right)=\mathbb{L}_{\omega} P_{\omega}\left(v_{b}, t_{b} \mid v_{a}, t_{a}\right)
$$

with the K-M operator

$$
\mathbb{L}_{\omega}=\sum_{n=1}^{\infty}\left(-\partial_{v}\right)^{n} K^{(n)}\left(v, t_{b a}\right)
$$

The expansion coefficients are obtained by analogy with (47) from the short-time limits

$$
\begin{gathered}
K^{(n)}(v, t)=\lim _{\tau \rightarrow 0} \frac{1}{n ! \tau} \int_{-\infty}^{\infty} \mathrm{d} v^{\prime}\left(v^{\prime}-v\right)^{n} P_{\omega}\left(v^{\prime}, t+\tau \mid v, t\right) \\
=\lim _{\tau \rightarrow 0} \frac{1}{n ! \tau}\left(\frac{\tau}{t_{b a}+\tau}\right)^{n} \int_{0}^{\infty} \mathrm{d} v^{\prime}\left(v^{\prime}-v\right)^{n} \omega\left(v^{\prime}, \tau\right) .
\end{gathered}
$$

A crucial observation for the further development is that due to the equality of the Kernel in the time evolution equation (53) and (49), the same K-M operator governs the time evolution of $\omega\left(v, t_{b a}\right)$ :

$$
\partial_{t_{b a}} \omega\left(v, t_{b a}\right)=\mathbb{L}_{\omega} \omega\left(v, t_{b a}\right)
$$

In Appendix A we show that this equation can also be derived directly from the K-M equations (44) and (45). 
The smearing procedure of the path integral is equivalent to replacing the pair of K-M equations (44) and (45) by the pair (44) and (56). This separates the dynamics of the smearing distribution from the dynamics of the transition amplitude. This may serve as a convenient starting point, for instance in quantum optics [24], in superstatistics [16], or in numerous option pricing models (see, e.g., Ref. [1] and citations therein).

For applications it is useful to remember Pawula's theorem [25], according to which the coefficients of the expansions of K-M operators are either all nonzero or, if there is a finite number of them, they can be nonzero only up to $n=2$. This follows from the necessary positivity of the probabilities. If one artificially truncates $D_{v}^{(n)}(x, t)$ in $(46)$ or $K^{(n)}(x, t)$ at some $n \geqslant 3$, then the ensuing transition probabilities always develop negative values, at least for sufficiently short times. This is the basic reason why phenomenological models for K-M operators go usually only up to $n=2$.

Consider such a truncated model. Then the K-M equations (44) and (56) reduce to the Fokker-Planck equations

$$
\begin{aligned}
\partial_{t} \omega(v, t) & =\mathbb{L}_{\omega}^{\mathrm{FP}} \omega(v, t), \\
\partial_{t_{b}} P_{v}\left(x_{b}, t_{b} \mid x_{a}, t_{a}\right) & =\mathbb{L}_{v}^{\mathrm{FP}} P_{v}\left(x_{b}, t_{b} \mid x_{a}, t_{a}\right),
\end{aligned}
$$

with

$$
\begin{aligned}
& \mathbb{L}_{\omega}^{\mathrm{FP}}=-\partial_{v} K^{(1)}(v, t)+\partial_{v}^{2} K^{(2)}(v, t), \\
& \mathbb{L}_{v}^{\mathrm{FP}}=-\partial_{x_{b}} D_{v}^{(1)}\left(x_{b}, t_{b}\right)+\partial_{x_{b}}^{2} D_{v}^{(2)}\left(x_{b}, t_{b}\right) .
\end{aligned}
$$

Furthermore, Eqs. (57) and (58) allow us to find two coupled stochastic processes described by the two coupled Itō stochastic differential equations

$$
\begin{aligned}
& \mathrm{d} x_{b}=D_{v}^{(1)}\left(x_{b}, t_{b}\right) \mathrm{d} t_{b}+\sqrt{2 D_{v}^{(2)}\left(x_{b}, t_{b}\right)} \mathrm{d} W_{1}, \\
& \mathrm{~d} v=K^{(1)}\left(v, t_{b a}\right) \mathrm{d} t_{b a}+\sqrt{2 K^{(2)}\left(v, t_{b a}\right)} \mathrm{d} W_{2} .
\end{aligned}
$$

Here $W_{1}\left(t_{b}\right)$ and $W_{2}\left(t_{b a}\right)$ are Wiener processes, i.e., Gaussian random walks.

\section{SIMPLE EXAMPLES}

To demonstrate usefulness of the superposition procedure we now discuss some important classes of $G(x)$-functions in Eq. (24). 
i.) We start with the trivial choice

$$
G(x)=e^{-a x+b}, \quad b \in \mathbb{R} ; a \in \mathbb{R}_{0}^{+},
$$

which gives

$$
\tilde{\omega}(\zeta, t)=e^{-a \zeta+b t}
$$

and consequently

$$
\omega(v, t)=e^{b t} \delta(v-a)
$$

By requiring that $\omega$ is normalized to 1 for any $t$ we have $b=0$, i.e., no smearing distribution. In this case the Hamiltonian $\bar{H}(p, x)=a H(p, x)$. Note that $a$ is basically the averaged value of $v$ over the $\delta$-function distribution.

ii.) A less trivial choice of $G(x)$ is

$$
G(x)=\left(\frac{a}{x+b}\right)^{c}, \quad a \in \mathbb{R}^{+} ; b, c \in \mathbb{R}_{0}^{+},
$$

which gives

$$
\tilde{\omega}(\zeta, t)=\left(\frac{a t}{\zeta+b t}\right)^{c t}
$$

leading thus to

$$
\omega(v, t)=\frac{1}{\Gamma(c t)}(a t)^{c t} e^{-b t v} v^{c t-1}
$$

Further restriction on the coefficients is obtained by requiring normalizability of $\omega(v, t)$. The normalization condition $F(0)=0$ can be fulfilled in two ways. Either we set $c=0$, in which case $\omega(v, t)=\delta(v)$ and $\bar{H}(p, x)=0$, or we assume that $a=b$. In the latter case

$$
\omega(v, t)=\frac{(b t)^{c t} v^{c t-1}}{\Gamma(c t)} e^{-b t v}
$$

i.e., it corresponds to the Gamma distribution $[4,10] f_{b t, c t}(v)$ which is of a particular importance in financial data analysis $[1,10,26]$ and superstatistics [27]. In the special case when $c=d b=d a$ and $b \rightarrow \infty$ we obtain that $\omega(v, t)=\delta(v-d)$. The Hamiltonian $\bar{H}$ associated with the distribution (69) reads

$$
\bar{H}(p, x)=\bar{v} b\left[\log \left(\frac{H(p, x)}{b}+1\right)\right]_{\mathrm{cl}},
$$


where $\bar{v}=c / b$ is the mean of $\omega(v, t)$. In particular, for $H=p^{2} / 2$ we obtain the smearing relation between path integrals

$$
\begin{aligned}
& \int_{x\left(t_{a}\right)=x_{a}}^{x\left(t_{b}\right)=x_{b}} \mathcal{D} x \mathcal{D} p e^{\int_{t_{a}}^{t_{b}} \mathrm{~d} \tau\left[i p \dot{x}-\bar{v} b \log \left(p^{2} / 2 b+1\right)\right]} \\
= & \int_{0}^{\infty} \mathrm{d} v \omega\left(v, t_{b a}\right) \int_{x\left(t_{a}\right)=x_{a}}^{x\left(t_{b}\right)=x_{b}} \mathcal{D} x \mathcal{D} p e^{\int_{t_{a}}^{t_{b}} \mathrm{~d} \tau\left(i p \dot{x}-v p^{2} / 2\right) .}
\end{aligned}
$$

Inserting for the path integral on the right-hand side the result (15), the integral over $v$ reads explicitly

$$
\int_{0}^{\infty} \mathrm{d} v \frac{\left(b t_{b a}\right)^{c t_{b a}} v^{c t_{b a}-1}}{\Gamma\left(c t_{b a}\right)} \sqrt{\frac{1}{2 \pi t_{b a} v}} e^{-b t_{b a} v} e^{-x_{b a}^{2} / 2 v t_{b a}}
$$

and yields

$$
K_{1 / 2-c t_{b a}}\left(2 \sqrt{2 b}\left|x_{b a}\right|\right) \frac{t_{b a}^{-3 / 2}}{\sqrt{\pi} \Gamma\left(c t_{b a}\right)}\left(\frac{2 \sqrt{2 b} t_{b a}}{\left|x_{b a}\right|}\right)^{1 / 2-c t_{b a}} .
$$

In Fourier space, the superposition (71) leads to a Tsallis distribution [10, 16, 27].

Another interesting consequence arises when we consider the ensuing Itō stochastic equations. To this end we use the Hamiltonian from Refs. [1, 10] which has the form $p^{2} / 2+i p(r / v-1 / 2)$, with $r$ being a constant. The corresponding drift and diffusion coefficients $D_{v}^{(1)}$ and $D_{v}^{(2)}$ are easily calculable giving

$$
D_{v}^{(1)}\left(x, t_{b}\right)=\left(r-\frac{v}{2}\right), \quad D_{v}^{(2)}\left(x, t_{b}\right)=\frac{v}{2} .
$$

The coefficients $K^{(n)}$ are

$$
\begin{aligned}
& K^{(1)}\left(v, t_{b a}\right)=\frac{1}{t_{b a}}\left(\frac{c}{b}-v\right)=\frac{1}{t_{b a}}(\bar{v}-v), \\
& K^{(n)}\left(v, t_{b a}\right)=\frac{1}{t_{b a}^{n}} \frac{c}{n b^{n}}, \quad n \geq 2 .
\end{aligned}
$$

Hence we find the two coupled Itō equations (61) and (62) in the form

$$
\begin{aligned}
& \mathrm{d} x_{b}=\left(r-\frac{v}{2}\right) \mathrm{d} t_{b}+\sqrt{v} \mathrm{~d} W_{1}, \\
& \mathrm{~d} v=\frac{1}{t_{b a}}(\bar{v}-v) \mathrm{d} t_{b a}+\frac{1}{t_{b a}} \sqrt{\frac{\bar{v}}{b}} \mathrm{~d} W_{2} .
\end{aligned}
$$

One may now view $x_{b}$ as a logarithm of a stock price $S$, and $v$ and $r$ as the corresponding variance and drift. If, in addition, we replace for large $t_{a b}$ the quantity $\sqrt{\bar{v}}$ with $\sqrt{v}$, the 
systems (76) reduces to

$$
\begin{aligned}
& \mathrm{d} S=r S \mathrm{~d} t_{b}+\sqrt{v} S \mathrm{~d} W_{1}, \\
& \mathrm{~d} v=\gamma(\bar{v}-v) \mathrm{d} t_{b a}+\varepsilon \sqrt{v} \mathrm{~d} W_{2},
\end{aligned}
$$

where $\gamma=1 / t_{b a}$ and $\varepsilon=1 /\left(b t_{b a}\right)$. The system of equations (77) constitute Heston's stochastic volatility model [26]. The parameters $\bar{v}, \gamma$ and $\varepsilon$ can be then interpreted as the long-time average, the drift of the variance and the volatility of the variance, respectively. Heston's model may be used in quantitative finance to evaluate, for instance, the price of options (for further reference on this model see, e.g., Ref. [1] and citations therein).

iii.) As a third example we consider

$$
G(x)=e^{-a \sqrt{x}}, \quad a \in \mathbb{R}^{+} .
$$

This implies

$$
\tilde{\omega}(\zeta, t)=e^{-a \sqrt{t \zeta}}
$$

and

$$
\omega(v, t)=\frac{a e^{-a^{2} \frac{t}{4 v}}}{2 \sqrt{\pi} \sqrt{\frac{v^{3}}{t}}} .
$$

Image function $\tilde{\omega}(\zeta, t)$ already fulfils the normalized condition $F(0)=0$. In the literature, (80) is known as the Weibull distribution of order 1 (e.g., Refs.[4, 32]) $\omega(v, t)=w\left(v ; 1, a^{2} t / 2\right)$. The smearing distribution (80) has also important applications in the so called inverse $\chi^{2}$ superstatistics [27]. Because integral over $v^{\alpha} \omega(v, t)$ does not exist for $\alpha>1 / 2$, the distribution (80) does not have moments. The Hamiltonian $\bar{H}(p, x)=a[\sqrt{H(p, x)}]_{c l}$. In particular, this implies an identity [29]

$$
\begin{gathered}
\int_{0}^{\infty} \mathrm{d} v w\left(v ; 1, \frac{t}{2}\right) \int_{\mathbf{x}\left(t_{a}\right)=\mathbf{x}_{a}}^{\mathbf{x}\left(t_{b}\right)=\mathbf{x}_{b}} \mathcal{D} \mathbf{x} \mathcal{D} \mathbf{p} e^{\int_{t a}^{t_{b}} \mathrm{~d} \tau\left[i \mathbf{p} \dot{\mathbf{x}}-v\left(\mathbf{p}^{2} c^{2}+m^{2} c^{4}\right)\right]} \\
=\int_{\mathbf{x}\left(t_{a}\right)=\mathbf{x}_{a}}^{\mathbf{x}\left(t_{b}\right)=\mathbf{x}_{b}} \mathcal{D} \mathbf{D} \mathbf{p} e^{\int_{t a}^{t_{b}} \mathrm{~d} \tau\left(i \mathbf{p} \dot{\mathbf{x}}-c \sqrt{\mathbf{p}^{2}+m^{2} c^{2}}\right)}
\end{gathered}
$$

The right-hand side represents the path integral for free relativistic particle in the NewtonWigner representation [30, 31]. Previously, this has been evaluated by group path integration [33]. With our smearing method, we can obtain the same result much faster by a direct 
calculation of the left-hand-side of (81). Due the the quadratic nature of the Hamiltonian we obtain immediately in $D$ space dimensions:

$$
\begin{aligned}
& \left.\int_{\mathbf{x}\left(t_{a}\right)=\mathbf{x}_{a}}^{\mathbf{x}\left(t_{b}\right)=\mathbf{x}_{b}} \mathcal{D} \mathbf{x} \mathcal{D} \mathbf{p} e^{\int_{t_{a}}^{t_{b}} \mathrm{~d} \tau\left(i \mathbf{p} \dot{\mathbf{x}}-c \sqrt{\mathbf{p}^{2}+m^{2} c^{2}}\right.}\right) \\
& =\int_{0}^{\infty} \mathrm{d} v \frac{e^{-\frac{t_{b a}}{4 v}}}{2 \sqrt{\pi} \sqrt{\frac{v^{3}}{t_{b a}}}} e^{-v m^{2} c^{4} t_{b a}-\frac{\mathbf{x}_{b a}^{2}}{4 v c^{2} t_{b a}}}\left(\frac{1}{4 v c^{2} \pi t_{b a}}\right)^{D / 2} \\
& =2 c t_{b a}\left(\frac{m \gamma}{2 \pi t_{b a}}\right)^{\frac{D+1}{2}} K_{\frac{D+1}{2}}\left(\frac{m c^{2} t_{b a}}{\gamma}\right),
\end{aligned}
$$

with $\gamma=\left(1+\mathbf{x}_{b a}^{2} / c^{2} t_{b a}^{2}\right)^{-1 / 2}, \mathbf{x}_{b a} \equiv \mathbf{x}_{b}-\mathbf{x}_{a}$. The result in Eq. (82) agrees, of course, with those of earlier authors [33].

If Eq. (82) is taken to the limit $m=0$, it reads

$$
\int_{\mathbf{x}\left(t_{a}\right)=\mathbf{x}_{a}}^{\mathbf{x}\left(t_{b}\right)=\mathbf{x}_{b}} \mathcal{D} \mathbf{x} \mathcal{D} \mathbf{p} e^{\int_{t_{a}}^{t_{b}} \mathrm{~d} \tau(i \mathbf{p} \dot{\mathbf{x}}-c|\mathbf{p}|)}=c t \Gamma\left(\frac{D+1}{2}\right)\left(\frac{\gamma^{2}}{\pi t^{2} c^{2}}\right)^{(D+1) / 2} .
$$

This is of a particular importance in econophysics as it can be directly related to a Lévy noise distribution of order 1, see, e.g. Ref. [1].

\section{CONCLUSIONS AND OUTLOOKS}

In this paper we have identified the most general class of continuous smearing distributions $\omega(v, t)$ defined on $\mathbb{R}^{+} \times \mathbb{R}^{+}$for which superpositions of Marcovian processes remain Markovian. This insight was used to rephrase the original problem of computing a complicated conditional probability $P\left(x_{b}, t_{b} \mid x_{a}, t_{a}\right)$ as a problem solving two coupled Kramers-Moyal equations, one for a simple basic distribution, and the other for the smearing distribution $\omega(v, t)$. If the associated Kramers-Moyal expansions are truncate after $n=2$, they become Fokker-Planck equations whose respective sample paths follow two coupled stochastic equations à la Itō. As an application of this decomposition procedure we have demonstrated how the log-normal fluctuations of a stock price with a Gamma smearing distribution of the variances lead to the celebrated Heston model of stochastic volatility.

As a second application we have shown that the ensuing relation between smeared path integrals and non-smeared ones permits one to solve exactly (and often quite fast) relatively large classes of path integrals. In this connection we have discussed in some detail two

important situations: path integrals arising in the framework of generalized statistics of 
Tsallis [28] and a world-line representation of the euclidean Newton-Wigner propagator. The latter situation can easily accommodate relativistic systems with gauge potentials via minimal coupling. Useful applications are expected in the field of world-line representations of effective actions $[14,35]$.

For simplicity we have considered smearing distributions for path integrals with only bosonic degrees of freedom. There is no problem in extending our procedure to path integrals with Grassmann (i.e., fermionic) variables, and to more general initial functional integrals. Such extensions may be useful in polymer physics, in particular in theory of self-avoiding chains, and in their field-theoretic treatments [1,36].

Our procedure can also be applied to quantum mechanics with only small modifications. There the $\mathrm{C}-\mathrm{K}$ relation is the composition law for time translation amplitudes reflecting the semigroup property of the evolution operator $e^{-i \int d t H}$. The smearing functions acts then upon probability amplitudes rather than conditional probabilities, in which case they may be complex rather than positive.

When the manuscript was finished we have became aware of Refs. [17, 27], devoted to superstatistics. There exists a conceptual overlap between the present paper and the above mentioned ones. In fact, practitioners in superstatistics can quickly benefit from our paper by substituting for our $v$ their intensive parameter $\beta$ and for our smearing distribution $\omega(v, t)$ their probability density $f(\beta)$. In superstatistical setting one could also call our smearing procedure as "the superstatistical average" (though our distributions are generally time dependent). In any case, it seems to us that important things are worth repeating several times using different framework and different words. For a more detailed discussion of the superstatistics paradigm, the reader is referred to Refs.[16, 17, 27].

\section{Acknowledgments}

We acknowledge discussions with Z. Haba and P. Haener. Work is partially supported by the Ministry of Education of the Czech Republic (research plan no. MSM 6840770039), by the Deutsche Forschungsgemeinschaft under grant Kl256/47. 


\section{Appendix A}

It is instructive to check that Eq. (58) can be derived directly from the original K-M equations (44) and (45) for $P_{v}\left(x_{b}, t_{b} \mid x_{a}, t_{a}\right)$ and $\bar{P}\left(x_{b}, t_{b} \mid x_{a}, t_{a}\right)$. This provides an important cross-check for the complete equivalence between both systems of K-M equations. To this end, we multiply $(44)$ by $\omega\left(v, t_{b a}\right)$ and integrate over $v$. This leads to

$$
\begin{gathered}
\partial_{t_{b}} \bar{P}\left(x_{b}, t_{b} \mid x_{a}, t_{a}\right)-\int_{0}^{\infty} \mathrm{d} v P_{v}\left(x_{b}, t_{b} \mid x_{a}, t_{a}\right) \partial_{t_{b a}} \omega\left(v, t_{b a}\right) \\
=\int_{0}^{\infty} \mathrm{d} v \omega\left(v, t_{b a}\right) \mathbb{L}_{v} P_{v}\left(x_{b}, t_{b} \mid x_{a}, t_{a}\right) .
\end{gathered}
$$

The first line has been rewritten using the chain rule for derivatives, while in the second line we have used the fact that $P_{v}\left(x_{b}, t_{b} \mid x_{a}, t_{a}\right)$ fulfills the K-M equation (44). We now insert in the second line a trivial unit integral $\int_{0}^{\infty} \mathrm{d} v^{\prime} \omega\left(v^{\prime}, \tau\right)=1$, where $\tau$ is a positive infinitesimal time. Then we subtract and add a term $\overline{\mathbb{L}} \bar{P}\left(x_{b}, t_{b} \mid x_{a}, t_{a}\right)$, i.e., the right-hand side of the $\mathrm{K}-\mathrm{M}$ equation (45). Thus the second line in (84) becomes

$$
\begin{aligned}
\int_{0}^{\infty} \mathrm{d} v & \int_{0}^{\infty} \mathrm{d} v^{\prime} \omega\left(v, t_{b a}\right) \omega\left(v^{\prime}, \tau\right)\left[\mathbb{L}_{v}-\mathbb{L}_{v^{\prime}}\right] P_{v}\left(x_{b}, t_{b} \mid x_{a}, t_{a}\right) \\
& +\overline{\mathbb{L}} \bar{P}\left(x_{b}, t_{b} \mid x_{a}, t_{a}\right) .
\end{aligned}
$$

Eq. (85) is a convenient short-hand version of more involved form where the $\tau \rightarrow 0$ limit acts both on $\omega(v, \tau)$ and $\mathbb{L}_{v}$. For instance,

$$
\cdots \int_{0}^{\infty} \mathrm{d} v^{\prime} \omega\left(v^{\prime}, \tau\right) \mathbb{L}_{v^{\prime}} \ldots
$$

actually means

$$
\ldots \sum_{k=1}^{\infty} \frac{1}{\tau k !} \int_{0}^{\infty} \mathrm{d} v^{\prime} \omega\left(v^{\prime}, \tau\right)\left(-\partial_{x_{b}}\right)^{k} \int_{-\infty}^{\infty} \mathrm{d} y\left(y-x_{b}\right)^{k} P_{v^{\prime}}\left(y, t_{b}+\tau, x_{b}, t_{b}\right) \ldots
$$

and similarly for the $\mathbb{L}_{v}$-term. With this in mind we now Taylor-expand $\mathbb{L}_{v^{\prime}}$ around $v$ and obtain

$$
\begin{gathered}
-\sum_{n=1}^{\infty} \frac{1}{n !} \int_{0}^{\infty} \mathrm{d} v \int_{0}^{\infty} \mathrm{d} v^{\prime} \omega\left(v, t_{b a}\right) \omega\left(v^{\prime}, \tau\right)\left(v^{\prime}-v\right)^{n}\left[\partial_{v}^{n} \mathbb{L}_{v}\right] P_{v}\left(x_{b}, t_{b} \mid x_{a}, t_{a}\right) \\
+\overline{\mathbb{L}} \bar{P}\left(x_{b}, t_{b} \mid x_{a}, t_{a}\right) .
\end{gathered}
$$

To compute the derivatives $\partial_{v}^{n} \mathbb{L}_{v}$ we use the representation (9) for a more general unsmeared Hamiltonian operator $H(\hat{p}, x)$ :

$$
P_{v}\left(x_{b}, t_{b} \mid x_{a}, t_{a}\right)=e^{-v t_{b a} \hat{H}\left(\hat{p}_{b}, x_{b}\right)} \delta\left(x_{b}-x_{a}\right)=e^{-v t_{b a}\left[\hat{H}^{\dagger}\left(\hat{p}_{a}, x_{a}\right)\right]^{*}} \delta\left(x_{b}-x_{a}\right),
$$


from which directly follows that

$$
\begin{aligned}
\partial_{v}^{n} P_{v}\left(y, t_{b}+\tau \mid x_{b}, t_{b}\right) & =(-\tau)^{n} \hat{H}^{n}(\hat{p}, y) e^{-v \tau \hat{H}(\hat{p}, y)} \delta\left(y-x_{b}\right) \\
& =(-\tau)^{n}\left[\left(\hat{H}^{\dagger}\right)^{n}\left(\hat{p}_{b}, x_{b}\right)\right]^{*} e^{-v \tau\left[\hat{H}^{\dagger}\left(\hat{p}_{b}, x_{b}\right)\right]^{*}} \delta\left(y-x_{b}\right) .
\end{aligned}
$$

Appearance of the term $\left[\hat{H}^{\dagger}(\hat{p}, x)\right]^{*}$ in the second lines is a consequence of the identity $\left\langle x_{b}|\hat{A}(\hat{p}, \hat{x})| x_{a}\right\rangle=\left\langle x_{a}\left|\hat{A}^{\dagger}(\hat{p}, \hat{x})\right| x_{b}\right\rangle^{*}$ which is valid for any operator $\hat{A}$. We can now simplify (88) with some algebra involving product rules of derivatives and $\delta$-functions. Abbreviating $\hat{H}(\hat{p}, x)$ by $\hat{H}_{x}$, this gives

$$
\begin{gathered}
-\sum_{n=1}^{\infty} \frac{1}{n ! \tau}\left(\frac{\tau}{t_{b a}+\tau}\right)^{n} \int_{0}^{\infty} \mathrm{d} v \int_{0}^{\infty} \mathrm{d} v^{\prime} \omega\left(v, t_{b a}\right) \omega\left(v^{\prime}, \tau\right)\left(v^{\prime}-v\right)^{n} \partial_{v}^{n} P_{v}\left(x_{b}, t_{b}+\tau \mid x_{a}, t_{a}\right) \\
+\overline{\mathbb{L}} \bar{P}\left(x_{b}, t_{b} \mid x_{a}, t_{a}\right) .
\end{gathered}
$$

In deriving the latter we have utilized the identity

$$
\int \mathrm{d} y\left(y-x_{b}\right)^{k}\left[\left(\hat{H}_{y}^{\dagger}\right)^{n}\right]^{*} e^{-v \tau\left(\hat{H}_{y}^{\dagger}\right)^{*}} \delta\left(y-x_{b}\right)=\int \mathrm{d} y \delta\left(y-x_{b}\right) \hat{H}_{y}^{n} e^{-v \tau \hat{H}_{y}}\left(y-x_{b}\right)^{k},
$$

and the fact that

$$
\hat{H}_{y}^{n} e^{-\tau v \hat{H}_{y}}=\left(\frac{-\partial_{\tau}}{v}\right)^{n} e^{-\tau v \hat{H}_{y}} .
$$

Extending for a moment the sum over $n$ in (88) to start from $n=0$, the relevant terms entering the calculation of $\left[\partial_{v}^{n} \mathbb{L}_{v}\right] P_{v}\left(x_{b}, t_{b} \mid x_{a}, t_{a}\right)$ in Eq. (91) have the form

$$
\begin{aligned}
& \sum_{k=0}^{\infty} \frac{\left(-\partial_{x_{b}}\right)^{k}}{k !} \delta\left(y-x_{b}\right) e^{-\tau v H_{y}}\left(y-x_{b}\right)^{k} e^{-t_{b a} v H_{x_{b}}} \delta\left(x_{b}-x_{a}\right) \\
& =\sum_{k=0}^{\infty} \frac{\left(-\partial_{x_{b}}\right)^{k}}{k !} \delta\left(y-x_{b}\right)[y(-i \tau)-y]^{k} e^{-t_{b a} v H_{y}} \delta\left(y-x_{a}\right) \\
& =e^{[y-y(-i \tau)] \partial_{x_{b}}} \delta\left(y-x_{b}\right) e^{-t_{b a} v H_{y}} \delta\left(y-x_{a}\right) .
\end{aligned}
$$

We now apply the shift operator $e^{[y-y(-i \tau)] \partial_{x_{b}}}$ to the $x_{b}$-variable in the $\delta$-function and cast the resulting $\delta\left(x_{b}-y(-i \tau)\right)$ into Dirac's bra-ket form

$$
\delta\left(x_{b}-y(-i \tau)\right)=\left\langle x_{b} \mid y(-i \tau)\right\rangle=\left\langle x_{b}\left|e^{-\tau v \hat{H}}\right| y\right\rangle
$$


If we now perform the integral over $y$ in (92) and use the fact that

$$
\left(\frac{-\partial_{\tau}}{v}\right)^{n}\left\langle x_{b}\left|e^{-v\left(\tau+t_{b a}\right) \hat{H}}\right| x_{a}\right\rangle=\left(\frac{-\partial_{v}}{t_{b a}+\tau}\right)^{n}\left\langle x_{b}\left|e^{-v\left(\tau+t_{b a}\right) \hat{H}}\right| x_{a}\right\rangle,
$$

we obtain the announced result (91). We now use the definition of the moments in the second line of Eq. (55), and the fact that the surface terms in the partial integrations over $v^{\prime}$ vanish due to the positivity of the real part of the Hamiltonian spectrum, to rewrite the first term in (91) as

$$
-\sum_{n=1}^{\infty} \int_{0}^{\infty} \mathrm{d} v\left(-\partial_{v}\right)^{n}\left[K^{(n)}\left(v, t_{b a}\right) \omega\left(v, t_{b a}\right)\right] P_{v}\left(x_{b}, t_{b} \mid x_{a}, t_{a}\right) .
$$

Remembering Eq. (54), this can be written as

$$
-\int \mathrm{d} v P_{v}\left(x_{b}, t_{b} \mid x_{a}, t_{a}\right) \mathbb{L}_{\omega} \omega\left(v, t_{b a}\right)
$$

If we now take into account the evolution equation (44), we obtain from Eq. (84) the equation

$$
\int_{0}^{\infty} \mathrm{d} v P_{v}\left(x_{b}, t_{b} \mid x_{a}, t_{a}\right) \partial_{t_{b a}} \omega\left(v, t_{b a}\right)=\int \mathrm{d} v P_{v}\left(x_{b}, t_{b} \mid x_{a}, t_{a}\right) \mathbb{L}_{\omega} \omega\left(v, t_{b a}\right) .
$$

This result must hold for any distribution $P_{v}\left(x_{b}, t_{b} \mid x_{a}, t_{a}\right)$, thus proving the $\mathrm{K}$-M equation (56) for $\omega(v, t)$.

\section{Appendix B}

Here we show how to define uniquely the classical Hamiltonian $F_{\mathrm{cl}}(H(p, x))$ if the initial Hamiltonian $H(p, x)$ depends not only on $p$ but also on $x$, This will be done with the help of the K-M equations.

We start with an observation that when the unsmeared K-M operator has no explicit time dependence, i.e., when

$$
\mathbb{L}_{v}\left(-\partial_{x}, x\right)=\sum_{n=1}^{\infty}\left(-\partial_{x}\right)^{n} D_{v}^{(n)}(x),
$$

then the unsmeared classical Hamiltonian $H_{\mathrm{cl}}(p, x)$ has the form

$$
H_{\mathrm{cl}}(p, x)=-\sum_{n=1}^{\infty}(-i p)^{n} D_{v}^{(n)}(x)
$$


provided we define the path integral by time slicing in the post-point form. This is done by rewriting the short-time matrix elements $\left\langle x_{n}|\exp (-\hat{H} \tau)| x_{n-1}\right\rangle$ as integrals $\int\left(\mathrm{d} p_{n} / 2 \pi\right)\left\langle x_{n} \mid p_{n}\right\rangle\left\langle p_{n}|\exp (-\hat{H} \tau)| x_{n-1}\right\rangle$ and defining the classical Hamiltonian via the identity

$$
\left\langle p_{n}|\exp (-\hat{H} \tau)| x_{n-1}\right\rangle \equiv e^{-\tau H_{\mathrm{cl}}\left(p_{n}, x_{n-1}\right)} e^{-i p_{n} x_{n-1}}
$$

Then to order $\mathcal{O}\left(\tau^{2}\right)$ the Hamiltonian $H_{\mathrm{cl}}(p, x)$ coincides with (99). Full discussion of this relation can be found in Ref. [1]. The K-M equation is basically a Schrödinger-type equation with the non-hermitian Hamiltonian $\hat{H}(p, x)=\sum_{n}(-i \hat{p})^{n} D_{v}^{(n)}(\hat{x})=-\mathbb{L}_{v}\left(-\partial_{x}, x\right)$.

Let us now see how the K-M equation looks for the smeared distribution. This will allow us to identify the smeared classical Hamiltonian $F_{\mathrm{cl}}(H)$. To this end we note that from the definition (47) the smeared K-M coefficients can be written as

$$
\begin{aligned}
D^{(n)}(x) & =\frac{1}{n !} \lim _{\tau \rightarrow 0} \frac{1}{\tau} \int_{0}^{\infty} \mathrm{d} v \omega(v, \tau) \int_{-\infty}^{\infty} \mathrm{d} y(y-x)^{n}\left\langle y\left|e^{-v \hat{H} \tau}\right| x\right\rangle \\
& =\frac{1}{n !} \lim _{\tau \rightarrow 0} \frac{1}{\tau} \int_{-\infty}^{\infty} \mathrm{d} y(y-x)^{n}\left\langle y\left|e^{-F(\hat{H}) \tau}\right| x\right\rangle .
\end{aligned}
$$

Furthermore, we can insert in front of $e^{-F(\hat{H}) \tau}$ a completeness relation $\int \mathrm{d} p|p\rangle\langle p|=\hat{\mathbf{1}}$, and make use of the fact that only terms up to order $\mathcal{O}(\tau)$ are relevant in $e^{-F(\hat{H}) \tau}$. This leads directly to

$$
\frac{1}{n !} \lim _{\tau \rightarrow 0} \frac{1}{\tau} \int_{-\infty}^{\infty} \mathrm{d} p \mathrm{~d} y(y-x)^{n}\langle y \mid p\rangle\left\langle p\left|e^{-F(\hat{H}) \tau}\right| x\right\rangle=\frac{1}{n !} \lim _{\tau \rightarrow 0} \frac{1}{\tau} \int_{-\infty}^{\infty} \frac{\mathrm{d} p \mathrm{~d} y}{2 \pi} y^{n} e^{i p y} e^{-F_{\mathrm{cl}}(H) \tau}
$$

Here $F_{\mathrm{cl}}(H)$ is obtained from $F(\hat{H})$ by using the commutator $[\hat{p}, x]=-i$ to move all $\hat{p}^{\prime}$ s to the left of all $x$ 's and dropping the hats. Eq. (102) can be further simplified if we write $F_{\mathrm{cl}}(H)=\sum_{n} p^{n} f_{n}(x)$ and the $y$-integral as the $n$-th derivative of $\delta$-function, i.e.

$$
\begin{aligned}
D^{(n)}(x) & =\frac{(-i)^{n}}{n !} \lim _{\tau \rightarrow 0} \frac{1}{\tau} \int_{-\infty}^{\infty} \mathrm{d} p e^{-\tau F_{\mathrm{cl}}(H)} \partial_{p}^{n} \delta(p) \\
& =\left.\frac{i^{n}}{n !} \lim _{\tau \rightarrow 0} \frac{1}{\tau} \partial_{p}^{n}\left[e^{-\tau F_{\mathrm{cl}}(H)}\right]\right|_{p=0}=(-i)^{n} f_{n}(x)
\end{aligned}
$$




\section{References}

[1] H. Kleinert, Path Integrals in Quantum Mechanics, Statistics, Polymer Physics and Financial Markets (World Scientific, Singapore 2004). We use the notation and conventions of this textbook.

[2] M. Kac, Probability and related Topics in Physical Sciences (Interscience, New York, 1959)

[3] R.P. Feynman and A.R. Hibbs, Quantum Mechanics and Path Integrals (McGraw-Hill, New York, 1965)

[4] W. Feller, An Introduction to Probability Theory and Its Applications, Vol. II (John Wiley, London, 1966)

[5] Z. Haba, Feynman Integral and Random Dynamics in Quantum Physics; A Probabilistic Approach to Quantum Dynamics (Kluwer, London, 1999)

[6] C.W. Gardiner, Handbook of Stochastic Methods for Physics, Chemistry and the Natural Sciences (Springer, Berlin, 1998)

[7] R. Friedrich and J. Peinke, Phys. Rev. Lett. 78 (1997) 863

[8] A.K. Myers-Beaghton and D.D. Vvedensky, J. Phys. A: Math. Gen. 22 (1989) L476

[9] D. Gross and C.M. Harris, Fundamentals of Queueing Theory (Wiley, London, 1998)

[10] P. Jizba, H. Kleinert and P. Haener, [arXiv:0708.3012]

[11] H. Kleinert and X.J. Chen, Physica A to be published [physics/0609209]

[12] G. Montagna, O. Nicrosini and N. Moreni, [arXiv:cond-mat/0202143]

[13] R.P. Feynman and H. Kleinert, Phys. Rev. A 34 (1986) 5080; R. Giachetti and V. Tognetti, Phys. Rev. B 33 (1986) 7647

[14] A.M. Polyakov, Gauge Fields and Strings (Harwood Academic Pub., Chur, 1987)

[15] A.L. Kholodenko, Ann. Phys. 202 (1990) 186

[16] C. Beck, Phys. Rev. Lett. 87 (2001) 180601; C. Beck and E.G.D. Cohen, Physica A 322 (2003) 267; G. Wilk and Z. Wlodarczyk, Phys. Rev. Lett. 84 (2000) 2770; F. Sattin, Physica A 338 (2004) 437

[17] H. Touchette and C.Beck, Phys. Rev. E 71 (2005) 16131

[18] see e.g., J. Aczél, Lectures on Functional Equations and their Applications (Academic Press, 
New York, 1966)

[19] G. Arfken, Mathematical Methods for Physicists, 3rd ed., (Academic Press, New York, 1985)

[20] E. Post, Trans. Amer. Math. Soc. 32 (1930) 723

[21] N. Bleistel and R.A. Handelsman, Asymptotic Expansions of Integrals (Dover Publications, New York, 1986)

[22] H. Kleinert and A. Chervyakov, Phys. Lett. B 464 (1999) 257.

[23] N. van Kampen, Stochastic Processes in Physics and Chemistry, (North Holland, London, 1982)

[24] H.J. Carmichael, Statistical Methods in Quantum Optics 1: Master Equations and FokkerPlanck Equations (Springer, London, 2003)

[25] R.F. Pawula, Phys. Rev. 162 (1967) 186

[26] S.L. Heston, Review of Financial Studies 6, 327 (1993).

[27] C. Beck, E.G.D. Cohen, H.L. Swinney, Phys. Rev. E 72 (2005) 56133

[28] C. Tsallis, J. Stat. Phys. 52 (1988) 479

[29] In the literature, Eq. (81) is often referred to as the Klein-Gordon kernel [34]. This name is misleading since a true kernel for the Klein-Gordon equation must include also the negative energy spectrum which itself reflects an existence of an independent charge-conjugated solution - antiparticle.

[30] T. Newton and E. Wigner, Rev. Mod. Phys. 21 (1949) 400

[31] J.B. Hartle and K.V. Kuchař, Phys. Rev. D 34 (1986) 2323

[32] W. Weibull, J. Appl. Mech.-Trans. ASME 18 (1951) 293

[33] E. Prugovečki, Nuovo Cimento A 61 (1981) 85; G. Junker, In Path Integrals from meV to MeV, Bangkok, 1989. Ed. V. Sa-yakanit et al. (World Scientific, Singapore, 1989), pp. 217.

[34] C. Grosche and F. Steiner, Handbook of Feynman Path Integrals (Springer, Berlin, 1998)

[35] C. Schubert, Physics Report 355 (2001) 73

[36] T.A. Vilgis, Physics Report 336 (2000) 167 